\title{
Lumen-apposing metal stents (LAMS) versus plastic stents for EUS-guided drainage of walled-off necrosis (WON) (LVPWON): study protocol for a multicenter randomized controlled trial
}

\author{
Hui-Yun Zhu ${ }^{1 \dagger}$, Pei Xie ${ }^{1,2+}$, Ying-Xiao Song ${ }^{1+}$, Zhao-Shen $\mathrm{Li}^{1,2,3,4^{*}}$, Zhen-Dong Jin ${ }^{1,2,3,4^{*}}$ and Yi-Qi Du ${ }^{1,2,3,4^{*}}$
}

\begin{abstract}
Background: Endoscopic ultrasonography (EUS)-guided drainage has become the first-line therapy for late peripancreatic fluid collection (PFC). Double pigtail plastic stents (DPPS) and lumen-apposing metal stents (LAMS) are commonly used for PFC drainage. Recently, a multi-institutional consensus on PFC drainage has recommended that LAMS should be the standard care for patients with walled-off necrosis (WON). However, given the poor quality of evidence, we aim to perform a large-scale randomized controlled trial to determine whether LAMS is superior to DPPS for WON drainage.

Methods/design: The study is an open-label, prospective, parallel-group, superiority, multicenter randomized controlled trial. Two hundred and fifty-six patients with WON who will attend 18 tertiary hospitals in China will be randomly allocated to the LAMS or DPPS group before the procedure. The primary endpoint is the clinical success at one month after drainage (reduction in the size of WON to $<2 \mathrm{~cm}$ ). Secondary endpoints include technical success, operation time, recurrence, adverse events, and secondary interventions.
\end{abstract}

Discussion: The LVPWON trial is designed to determine whether LAMS is effective, safe, and superior to DPPS for WON drainage.

Trial registration: ClinicalTrials.gov, NCT03027895. Registered on 14 January 2017.

Keywords: LAMS, DPPS, WON, Trial, EUS

\section{Background}

Walled-off necrosis (WON) is a type of pancreatic fluid collection (PFC) that develops in the setting of acute or chronic pancreatitis, trauma, or pancreatic duct obstruction [1]. WON, a delayed complication of necrotizing pancreatitis usually occurring $>4$ weeks following the onset of pancreatitis, is PFC surrounded by a radiologically identifiable capsule containing both solid and liquid

\footnotetext{
* Correspondence: 15026921681@163.com; jinzd66@126.com; duyiqi006@126.com

${ }^{\dagger}$ Hui-Yun Zhu, Pei Xie and Ying-Xiao Song contributed equally to this work. ${ }^{1}$ Department of Gastroenterology, Changhai Hospital, Second Military Medical University/ Naval Medical University, 168 Changhai Road, Shanghai 200433, China

Full list of author information is available at the end of the article
}

components [1]. Most WONs are asymptomatic and resolve spontaneously. However, drainage is necessary when it becomes symptomatic or infected, or increases in size over the course of the illness.

In the past decade, the treatment of symptomatic WON has evolved from surgical to endoscopic necrosectomy. Endoscopic treatment has a reduced proinflammatory response compared to surgery for the treatment of WONs [2]. It is associated with lower rates of pancreatic fistula formation and shorter hospital stays [3, 4]. Endoscopic ultrasonography (EUS)-guided drainage has high technical and clinical success and is associated with low adverse events (AEs) making it the optimal drainage approach for WONs $[5,6]$. 
Double pigtail plastic stents (DPPS) is the standard choice for pancreatic pseudocyst drainage with $>90 \%$ technical and clinical success rates [5]. DPPS has also been used for WON drainage [7]. Recently, a novel lumen-apposing metal stent (LAMS) with a larger luminal diameter $(\geq 10 \mathrm{~mm}$ ) has been successfully used for EUS-guided drainage of PFC [8]. A multi-institutional consensus made by 22 expert endosonographers recommended that LAMS should be the standard of care for WON drainage [9].

However, the safety of LAMS is still controversial. Some studies have reported that LAMS is superior to DPPS in terms of overall treatment efficacy ( $90 \%$ vs $81 \%$ ) and a significantly lower number of procedures (2.2 vs 3.6) [10]. LAMS includes single-step deployment and has an anti-migration structure $[11,12]$. Other studies have proposed that LAMS was associated with a significantly higher rate of bleeding compared with DPPS [13-15]. Moreover, high quality of evidence with regards to the efficacy and safety of LAMS for WON drainage in Chinese patients is lacking. Given the recent widespread use of LAMS in the management of WON, we designed this open-label, prospective, parallel-group, superiority, multicenter randomized controlled trial (RCT) to investigate whether LAMS is superior to DPPS for WON drainage.

\section{Methods/design}

\section{Design}

The LVPWON trial is an open-label, prospective, parallelgroup, superiority, multi-center RCT designed to determine whether LAMS is effective and safe in the EUSguided drainage of WON and superior to DPPS. Patients with WON admitted at 18 tertiary hospitals in China will be randomly allocated to the LAMS or DPPS group before the procedure. The study protocol has been approved by the Institutional Review Board and Ethics Committee of Shanghai Changhai Hospital. Recommendations for Interventional Trial (SPIRIT) Checklist have been provided (see Additional file 1).

\section{Study population}

This prospective study will be performed at the $\mathrm{Na}$ tional Clinical Research Center for Digestive Diseases and 18 tertiary hospitals in China. All adult patients admitted with WON will be assessed for eligibility during their hospital admission. If patients are found to have solid debris in the PFC and fulfill all inclusion and exclusion criteria, they will be randomized (at a 1:1 ratio) to the LAMS or DPPS group after obtaining signed informed consent (Fig. 1). In this experiment, randomization will be performed using the Interactive Web-Respond System (IWRS).

The inclusion and exclusion criteria are listed in Table 1. All patients (aged $\geq 18$ years) with WON

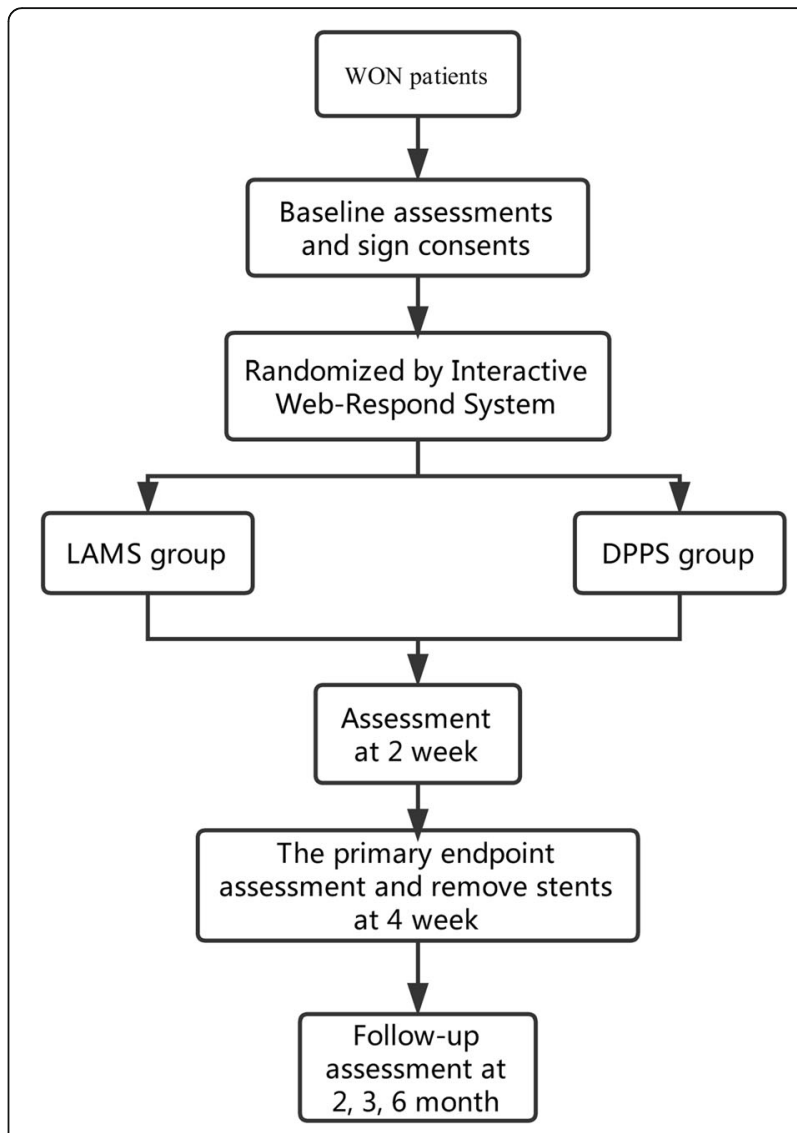

Fig. 1 Research flow chart

Table 1 Inclusion and exclusion criteria

Inclusion criteria

Aged $18-80$ years

Individual with WON confirmed by CT and MRCP

The size of WON $\geq 6 \mathrm{~cm}$ and located adjacent to the gastric wall

Participants has symptoms related to the WON

Written informed consent obtained

Exclusion criteria

Aged $<18$ years or $>80$ years

Individual cannot accept the endoscopic procedure

The distance between the stomach and the wall of the $W O N \geq 1 \mathrm{~cm}$ Participant has blood coagulation dysfunction (platelet count $<50 \times$ 109/L or INR > 1.5)

Allergic to nickel titanium

Suffering from severe lung or heart disease

Pregnant and lactating women and those who are about to become pregnant soon

Any other factors that are not suitable for inclusion or influence the individual's participation in the study judged by researchers

WON walled-off necrosis, INR international normalized ratio 
requiring drainage and fulfilling the inclusion criteria will be eligible for enrollment. Investigators will inform the patients about the trial procedure. Patients will independently choose to participate in the trial and sign the informed consent. Any participant has the right to opt out of this trial at any time. Contraindications for EUS-guided drainage of WON will be determined by endoscopists or anesthesiologists before the procedure. CT images obtained from the 18 hospitals will be uniformly read by Changhai Hospital.

\section{Treatment protocol}

Before the procedure, if an individual meets the inclusion and exclusion criteria, the investigator will report this to the leader unit (Shanghai Changhai Hospital) and the participant will be randomized to receive either LAMS (Fig. 2) or DPPS for WON drainage. Randomization will occur in a 1:1 fashion with a random number table generated by IWRS, making it possible for all patients from different hospitals to be randomly assigned to each group.

The operation will be performed under mild sedation, monitored under anesthesia care or under general anesthesia, and prophylactic antibiotics will be used when appropriate. EUS-guided drainage will be performed by expert endosonographers after unified standard operation training. First, the position and size of the WON will be determined by endoscopic ultrasonography and the appropriate puncture point will be selected. Next, a 19G puncture needle will be inserted into the WON via the endoscope. Under fluoroscopy guidance, a 0.035 in. $(1$ in. $=0.025 \mathrm{~m})$ yellow zebra guide wire will be inserted and coiled into the WON cavity. A Wilson COOK cystotome electric

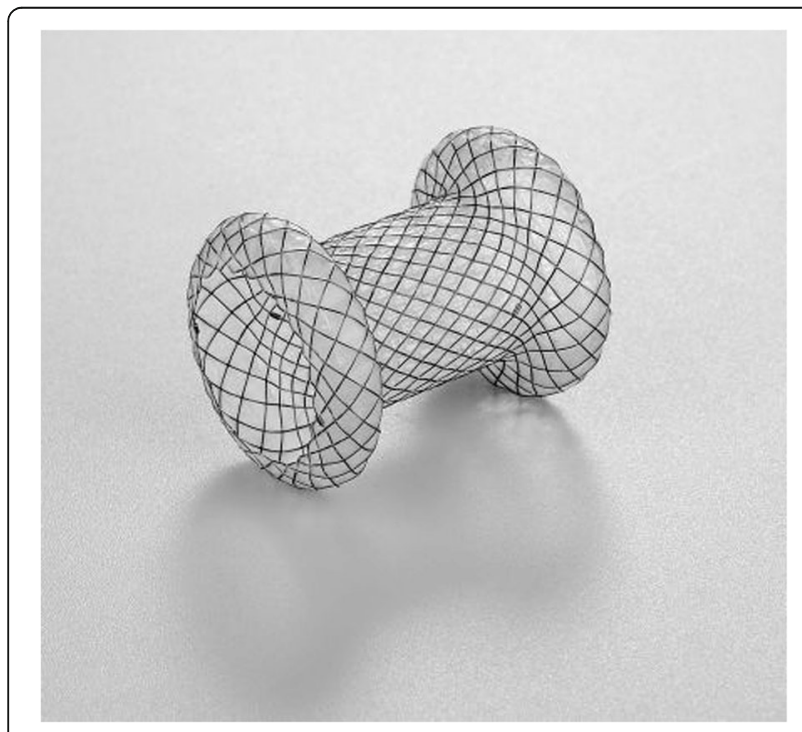

Fig. 2 Lumen-apposing metal stent (Micro-TechCo. Ltd., Nanjing, China) knife will be placed along the guide wire to cut the wall of stomach and the wall of the WON. Balloon expansion will be used if necessary. Subsequently, after drainage of the liquid content of the WON in to the stomach, LAMS or DPPSs would be placed to maintain the opening.

\section{Data collection and follow-up}

Investigators will save a recording of the operation and note the procedure time. After EUS-guided drainage, patients will be sent back to the ward. Patients will be monitored for $3 \mathrm{~h}$ and $24 \mathrm{~h}$ serum amylase after the operation and prophylactic antibiotics will be administered postoperatively. Detailed data information can be found in the SPIRIT figure (Fig. 3). After the LAMS is implanted, if the WON needs to be cleaned, direct endoscopic necrosectomy (DEN) will be performed. Stents will be removed one month after implantation. If the clinical success criterion is not met at the onemonth follow-up, the patient will be provided with further treatment that will be decided based on their individual needs. All individuals who participated in the trial will be followed up at one, three, and six months post operation, with a final follow-up to occur 12 months after the end of the trial for CT evaluation. The above data will be collected into case report forms (CRFs). Stent-related AEs and management will also be recorded and reported in the CRFs. Finally, CRFs will be summarized by the $\mathrm{Na}$ tional Clinical Research Center for Digestive Diseases.

\section{Outcomes}

The primary outcome is the clinical success rate at one month after drainage. Clinical success is defined as reduction in the size of $\mathrm{WON}$ to $<2 \mathrm{~cm}$. Within one month of the drainage, if the patient needs to change the treatment method (such as the additional placement of nasal cyst drainage tube; or the DPPS removed and replaced with a LAMS), it will be recorded as drainage failure. The secondary outcome is technical success, operation time, recurrence, AEs, and secondary interventions. The definitions of the primary and secondary outcomes are presented in Table 2.

\section{Statistical considerations}

Sample size The sample size calculation is based on a literature review and conference discussion regarding the clinical success rate of LAMS or DPPS drainage for WON. The LVPWON trial is a superiority trial in which the sample size calculation was based on the assumption that the incidence of the primary endpoint with LAMS and DPPS is $90 \%$ and $75 \%$, respectively. Assuming a one-side alpha (type I error) of 0.025 and a power of $85 \%$ (beta, a type II error, was taken as 0.15 ), we used the following formula to calculate sample size and found 


\begin{tabular}{|c|c|c|c|c|c|c|c|}
\hline \multirow{2}{*}{ Research stages } & \multirow{2}{*}{$\begin{array}{c}\text { Baseline }^{0} \\
-7 \sim 0 \mathrm{~d}\end{array}$} & \multirow{2}{*}{$\begin{array}{c}\begin{array}{c}\text { Interventional } \\
\text { treatment }\end{array} \\
\text { During operation }\end{array}$} & \multirow{2}{*}{$\begin{array}{c}\text { Follow-up } \\
14 \pm 3 d\end{array}$} & \multirow{2}{*}{$\begin{array}{c}\text { Stent removement } \\
33 \pm 3 \mathrm{~d}\end{array}$} & \multicolumn{3}{|c|}{ Follow-up period } \\
\hline & & & & & $60 \pm 7 d$ & $90 \pm 7 d$ & $180 \pm 7 d$ \\
\hline Informed consent & $\mathrm{x}$ & & & & & & \\
\hline demographics & $\mathrm{x}$ & & & & & & \\
\hline $\begin{array}{c}\text { Current and past medical } \\
\text { history }\end{array}$ & $\mathrm{x}$ & & & & & & \\
\hline Vital signs & $\mathrm{x}$ & $\mathrm{x}$ & & $\mathrm{X}$ & & & \\
\hline $\begin{array}{c}\text { Inclusion and exclusion } \\
\text { criteria }\end{array}$ & $\mathrm{x}$ & & & & & & \\
\hline Randomization & $\mathrm{X}$ & & & & & & \\
\hline Operation records & & $\mathrm{X}$ & & & & & \\
\hline Stent implantation & & $\mathrm{x}$ & & & & & \\
\hline Stent removal ${ }^{1}$ & & & & $\mathrm{x}$ & & & \\
\hline Secondary intervention & & $\mathrm{X}$ & & & & & \\
\hline Adverse event & & $\mathrm{x}$ & $\mathrm{x}$ & $\mathrm{x}$ & $\mathrm{X}$ & $\mathrm{x}$ & $\mathrm{x}$ \\
\hline Blood routine $^{2}$ & $\mathrm{X}$ & & & $\mathrm{x}$ & & & \\
\hline Liver and kidney function ${ }^{3}$ & $\mathrm{x}$ & & & $\mathrm{x}$ & & & \\
\hline Coagulation function ${ }^{4}$ & $\mathrm{x}$ & & & $\mathrm{X}$ & & & \\
\hline Serum amylase $e^{5}$ & $\mathrm{x}$ & $\mathrm{x}$ & & & & & \\
\hline MRCP & $\mathrm{x}$ & & & & & & \\
\hline $\mathrm{CT}^{6}$ & $\mathrm{x}$ & & & $\mathrm{x}$ & & $\mathrm{X}$ & $\mathrm{x}$ \\
\hline The primary endpoint ${ }^{7}$ & & & & $\mathrm{x}$ & & & \\
\hline Secondary endpoints ${ }^{8}$ & & & & $\mathrm{X}$ & & & \\
\hline Combination therapy & $\mathrm{x}$ & $\mathrm{x}$ & $\mathrm{x}$ & $\mathrm{x}$ & $\mathrm{x}$ & $\mathrm{X}$ & $\mathrm{X}$ \\
\hline
\end{tabular}

Fig. 3 Schedule of enrollment, follow-up, and assessments. Basline ${ }^{0}$ : screening of laboratory tests can be accepted the previous week in our hospital inspection report. Stent removal': time from stenting to postoperative withdrawal (33 \pm 3 days). Blood routine ${ }^{2}$ : hemoglobin (HGB), erythrocyte count $(\mathrm{RBC})$, platelet count $(\mathrm{PLT})$, leukocyte count (WBC), neutrophil percentage (\%), lymphocyte percentage (\%). Liver and kidney function ${ }^{3}$ : alanine aminotransferase (ALT), aspartate aminotransferase (AST), total bilirubin, direct bilirubin (DBIL), indirect bilirubin (IBIL), alkaline phosphatase (ALP), glutamyl transferase (GGT), blood urea nitrogen (BUN), creatinine (Cr). Coagulation function ${ }^{4}$ : prothrombin time (PT), fibrinogen (FIB), activated partial thromboplastin time (APTT), thrombin time (TT). Serum amylase ${ }^{5}$ : assessed $3 \mathrm{~h}$ and $24 \mathrm{~h}$ after the operation. $\mathrm{CT}^{6}$ : preoperative and postoperative enhanced $C T$ examination, 3 months and 6 months postoperative $C T$ examination. The primary endpoint ${ }^{7}$ : immediate success rate of surgery, drainage success rate 1 month after surgery. Secondary endpoints ${ }^{8}$ : the incidence of clinical complications, operation time, the recurrence rate of pancreatic pseudocyst within 1 month and 1 month after the operation, and secondary interventions

that 256 patients (128 per condition) would be necessary, including a possible dropout rate of $10 \%$.

$$
n=\frac{\left[\mu_{1-\alpha} \sqrt{2 \bar{p}(1-\bar{p})}+\mu_{1-\beta} \sqrt{p_{T}\left(1-p_{T}\right)+p_{C}\left(1-p_{C}\right)}\right]^{2}}{\left(p_{T}-p_{C}\right)^{2}}
$$

In this formula, $p_{T}$ represents expected success rate of the LAMS group, $p_{C}$ indicates expected success rate of the DPPS group, $\bar{p}$ is average success rate of the two groups, and $\mu$ represents the quantile of standard normal distribution.

Data management Patient characteristics will be recorded in a CRF. An electronic data collection system (electronic data capture [EDC]) will be used to complete the trial data collection. The EDC system has been rigorously tested to fully meet the requirements of the "Quality Management Practice for Medical Device Clinical Trials" and "Technical Guidelines for Clinical Trial Data Management Work." Before the system is officially launched, training tests will be conducted on relevant users to ensure that the system meets the trial requirements. After the official launch, related personnel will receive the account number and password. The account is bound to the user's role and permissions: the user must properly maintain the account information, not inform others about the account information, and exercise appropriate rights for others. Clinical auditors will monitor the clinical trial center's work at least once a month. This trial has an interim analysis once half of the required number of the patients have been enrolled, 
Table 2 Definitions of the primary and secondary outcomes

\begin{tabular}{|c|c|}
\hline Outcomes & Description \\
\hline Clinical success & The diameter of the WON $\leq 2 \mathrm{~cm}$ \\
\hline Technical success & LAMS or DPPS deployed successfully \\
\hline Operation time & $\begin{array}{l}\text { Time duration from the beginning to the end of the EUS-guided stent } \\
\text { implantation procedure }\end{array}$ \\
\hline Recurrence & $\begin{array}{l}\text { After successful drainage, cyst reappears on imaging examinations with } \\
\text { symptoms requiring intervention }\end{array}$ \\
\hline \multicolumn{2}{|l|}{ Adverse event } \\
\hline Perforation & Imaging manifests as pneumoperitoneum with peritoneal irritation syndrome \\
\hline Bleeding & Any bleeding that requires intervention, blood transfusion, and hospital observation \\
\hline Suprainfection & $\begin{array}{l}\text { Postoperative fever, increased inflammatory index (CRP, PCT), or positive } \\
\text { blood culture }\end{array}$ \\
\hline Occlusion & The stent is filled with tissue or debris \\
\hline Migration & The stent is completely or partially displaced into the WON or stomach \\
\hline Others & Adverse events that occurred in the trial but not described above \\
\hline Secondary intervention & Any endoscopic operation after stent is placed \\
\hline
\end{tabular}

CRP C-reactive protein, $P C T$ procalcitonin

during which the data will be analyzed. If a serious $A E$ is identified, the study will be terminated immediately.

Statistical analysis plan Categorical data will be described using frequency and composition ratios. Continuous data will be described using mean, standard deviation, maximum, minimum, and median, as well as 25 th and 75 th quantiles. Baseline demographic analysis based on the descriptive analysis, the likelihood ratio $X^{2}$ test will be used for the comparison between the categorical data groups. Fisher's exact probability method will be used when theoretical frequency of cells exceeding $25 \%$ is $<5$. Normally distributed continuous datasets will be compared using a group $t$-test. For non-normally distributed continuous data, Wilcoxon rank sum test will be used for comparison between groups. This is a multi-center study. The test conditions of each center are not identical (such as operators, etc.) and the outcomes may be different. For the primary outcome, comparison between groups will be performed using the $\mathrm{CMH}$ (Cochran-MantelHaensel) $\chi^{2}$ analysis to adjust for the central effect. The differences between the success rates of the groups and their 95\% confidence intervals (CI) will also be estimated. AEs will be described by the number and incidence of AEs; the likelihood ratios between groups will be compared using the $\chi^{2}$ test or Fisher's exact probability test. Major investigators will have access to the final trial dataset and the statistician involved will be blinded to the treatment assignments. Statistical analysis will be performed with two-sided tests at a level of significance of 0.05 (except where specified). Data analysis will be conducted using SAS 9.4 statistical software.

\section{Discussion}

The LVPWON trial has been designed to answer the question of whether LAMS is superior to DPPS for EUS-guided drainage of WON with regards to the clinical success rate and the incidence of AEs. We also want to prospectively investigate the risk factors for success of treatment and complications of EUS-guided drainage, which will provide a reference for the clinical treatment of WON.

EUS-guided drainage has been maturely applied in late PFC with well-defined inflammatory walls [7, 16-18]. Stents used for drainage are diverse. DPPS used in the management of PFC was first reported in the 1980s [19]. Subsequently, self-expanding metal stents (SEMS) and double-flanged LAMS have become the most popular drainage stents for PFC $[8,11,12]$.

Due to their small diameter, several DPPSs are needed to achieve adequate drainage of a WON. Metal stents that have a larger diameter are theoretically considered superior to DPPS as they allow for the possibility of WON debridement. A previous study directly comparing DPPS and LAMS demonstrated that the latter had a success rate of $90 \%$, which was higher than the former, and a significantly fewer number of procedures were required with LAMS for WON resolution [10]. In terms of AEs, stent occlusion seems more likely to occur in WON treated with DPPS [10]. Furthermore, new technology associated with LAMS enhance the drainage effect [20]. The procedure of LAMS deployment has become much easier and more economical.

However, with the increasing use of LAMS for WON drainage, LAMS-related bleeding has been more frequently reported [13-15]. In combined endoscopic and percutaneous drainage for symptomatic WON, LAMS 
did not reduce the time to percutaneous drain removal and was not associated with fewer AEs [21]. Furthermore, LAMS is substantially more expensive than DPPS.

Recently, a consensus guideline formulated by the Asian EUS group RAND/UCLA expert panel raised the issue of stent selection [5]. There are currently no randomized data addressing how LAMS compare with DPPS for WON drainage. An ongoing RCT observed serious LAMS-related AEs, including delayed bleeding in $50 \%$ of patients $(6 / 12)$, buried stent syndrome, and biliary stricture [14]. However, our team had analyzed our center's experience and determined that the use of LAMS is safe [22].

The LVPWON trial was initiated by Changhai Hospital, which is a National Clinical Research Center for Digestive Diseases. This is the largest prospective, open-label, parallel-group, superiority, multi-center RCT to address the appropriate selection of the stent for WON drainage. The trial includes 18 Chinese tertiary hospitals. Despite discrepancies in diagnostic and operational skill levels at different centers, as the research initiator, Changhai Hospital will provide technical training. Only experienced endosonographers will perform drainage procedures. The trial will also adopt "centralized readings" to reduce the heterogeneity between centers. Given the differences in the type of stent that will be placed, endoscopists and patients will not be blinded to the treatment allocation. However, the outcomes of the trial are unlikely to be affected by the patient's psychological factors.

In conclusion, the major focus of the LVPWON trial is to prospectively compare the efficacy and safety of LAMS and DPPS in EUS-guided drainage of WON and to identify the risk factors associated with LAMS-related complications, thus further benefiting WON patients treated with LAMS.

\section{Trial status}

This multicenter RCT is expected to begin enrolling patients on 30 September 2018. Protocol version number: V 2.0, 2017-10-10. The approximate date when recruitment will be completed is 30 September 2019.

\section{Additional file}

Additional file 1: SPIRIT checklist. (DOC $118 \mathrm{~kb}$ )

\section{Abbreviations}

Cl: Confidence interval; CMH: Cochran-Mantel-Haensel; CRF: Case report form; CRP: C-reactive protein; DEN: Direct endoscopic necrosectomy; DPPS: Double pigtail plastic stents; EDC: Electronic data capture; EUS: Endoscopic ultrasonography; IWRS: Interactive Web-Respond System; LAMS: Lumen-apposing metal stents; LVPWON: LAMS vs DPPS trial; PCT: Procalcitonin; PFC: Pancreatic fluid collection; SEMS: Self-expanding metal stent; WON: Walled-off necrosis
Acknowledgements

We thank Dr. Li Wei for the sample size calculation.

\section{Funding}

Support for this study came from the National Natural Science Foundation of China (Grant no. 81770643 to YQD), Shanghai Excellent Discipline Leader Training Program (Grant no. $2017 B R 048$ to YQD), Innovative Fund for PhD granted by Second Military Medical University/ Naval Medical University (to HYZ). The funding bodies have no role in the trial design or interpretation of the data.

\section{Authors' contributions}

HYZ drafted the manuscript. HYZ, PX, and YXS participated in the enrollment of patients of the study. HYZ and PX registered the study. ZDJ, ZSL, and YQD participated in the design of the study. ZDJ and YQD conceived the project, designed the study, revised the manuscript, and approved the final submission. All authors read and approved the final manuscript.

\section{Ethics approval and consent to participate}

Ethical approval has been obtained from Changhai Institutional Review Board (CHEC2017-192). Written informed consent will be obtained from each patient before randomization. Any subsequent amendments of the protocol need to be approved by the relevant ethical bodies before implementation.

\section{Consent for publication}

Not applicable.

\section{Competing interests}

The authors declare that they have no competing interests.

\section{Publisher's Note}

Springer Nature remains neutral with regard to jurisdictional claims in published maps and institutional affiliations.

\section{Author details}

'Department of Gastroenterology, Changhai Hospital, Second Military Medical University/ Naval Medical University, 168 Changhai Road, Shanghai 200433, China. ${ }^{2}$ Digestive Endoscopy Center, Changhai Hospital, Second Military Medical University/ Naval Medical University, 168 Changhai Road, Shanghai 200433, China. ${ }^{3}$ Shanghai Institute of Pancreatic Diseases, Shanghai, China. ${ }^{4}$ National Clinical Research Center of Digestive Diseases, Shanghai, China.

Received: 28 March 2018 Accepted: 3 September 2018 Published online: 11 October 2018

\section{References}

1. Banks PA, Bollen TL, Dervenis C, Gooszen HG, Johnson CD, Sarr MG, et al. Classification of acute pancreatitis--2012: revision of the Atlanta classification and definitions by international consensus. Gut. 2013;62:102-11.

2. Bakker OJ, van Santvoort HC, van Brunschot S, Geskus RB, Besselink MG, Bollen $\mathrm{TL}$, et al. Endoscopic transgastric vs surgical necrosectomy for infected necrotizing pancreatitis: a randomized trial. JAMA. 2012;307:1053-61.

3. van Brunschot S, van Grinsven J, van Santvoort HC, Bakker OJ, Besselink MG, Boermeester MA, et al. Endoscopic or surgical step-up approach for infected necrotising pancreatitis: a multicentre randomised trial. Lancet. 2018:391:51-8.

4. Varadarajulu S, Bang JY, Sutton BS, Trevino JM, Christein JD, Wilcox CM. Equal efficacy of endoscopic and surgical cystogastrostomy for pancreatic pseudocyst drainage in a randomized trial. Gastroenterology. 2013;145:583-90 e1.

5. Teoh AYB, Dhir V, Kida M, Yasuda I, Jin ZD, Seo DW, et al. Consensus guidelines on the optimal management in interventional EUS procedures: results from the Asian EUS group RAND/UCLA expert panel. Gut. 2018;67:1209-28.

6. Zhu H, Jiang F, Zhu J, Du Y, Jin Z, Li Z. Assessment of morbidity and mortality associated with EUS-guided FNA for pancreatic cystic lesions: a system review and meta-analysis. Dig Endosc. 2017;29:667-75.

7. Shekhar C, Maher B, Forde C, Mahon BS. Endoscopic ultrasound-guided pancreatic fluid collections' transmural drainage outcomes in 100 consecutive cases of pseudocysts and walled off necrosis: a single-centre experience from the United Kingdom. Scand J Gastroenterol. 2018;53:611-15. 
8. Walter D, Will U, Sanchez-Yague A, Brenke D, Hampe J, Wollny H, et al. A novel lumen-apposing metal stent for endoscopic ultrasound-guided drainage of pancreatic fluid collections: a prospective cohort study. Endoscopy. 2015:47:63-7.

9. Sun S, Adler D, Guo J, Saftoiu A, Vilmann P, Fusaroli P, et al. A multiinstitutional consensus on how to perform endoscopic ultrasound-guided peri-pancreatic fluid collection drainage and endoscopic necrosectomy. Endoscopic Ultrasound. 2017;6:285.

10. Siddiqui AA, Kowalski TE, Loren DE, Khalid A, Soomro A, Mazhar SM, et al. Fully covered self-expanding metal stents versus lumen-apposing fully covered self-expanding metal stent versus plastic stents for endoscopic drainage of pancreatic walled-off necrosis: clinical outcomes and success. Gastrointest Endosc. 2017:85:758-65.

11. Shah RJ, Shah JN, Waxman I, Kowalski TE, Sanchez-Yague A, Nieto J, et al. Safety and efficacy of endoscopic ultrasound-guided drainage of pancreatic fluid collections with lumen-apposing covered self-expanding metal stents. Clin Gastroenterol Hepatol. 2015;13:747-52.

12. Sharaiha RZ, Tyberg A, Khashab MA, Kumta NA, Karia K, Nieto J, et al. Endoscopic therapy with lumen-apposing metal stents is safe and effective for patients with pancreatic walled-off necrosis. Clin Gastroenterol Hepatol. 2016;14:1797-803.

13. Lang GD, Fritz C, Bhat T, Das KK, Murad FM, Early DS, et al. EUS-guided drainage of peripancreatic fluid collections with lumen-apposing metal stents and plastic double-pigtail stents: comparison of efficacy and adverse event rates. Gastrointest Endosc. 2018:87:150-7.

14. Bang JY, Hasan M, Navaneethan U, Hawes R, Varadarajulu S. Lumenapposing metal stents (LAMS) for pancreatic fluid collection (PFC) drainage: may not be business as usual. Gut. 2017;66:2054-6.

15. Brimhall B, Han S, Tatman PD, Clark TJ, Wani S, Brauer B, et al. Increased incidence of Pseudoaneurysm bleeding with lumen-apposing metal stents compared to double pigtail plastic stents in patients with peripancreatic fluid collections. Clin Gastroenterol Hepatol. 2018;16:1521-8.

16. Watanabe Y, Mikata R, Yasui S, Ohyama H, Sugiyama H, Sakai Y, et al. Shortand long-term results of endoscopic ultrasound-guided transmural drainage for pancreatic pseudocysts and walled-off necrosis. World J Gastroenterol. 2017;23:7110-8.

17. Dhir V, Adler DG, Dalal A, Aherrao N, Shah R, Maydeo A. Early removal of biflanged metal stents in the management of pancreatic walled-off necrosis: a prospective study. Endoscopy. 2018;50:597-605.

18. Rinninella E, Kunda R, Dollhopf M, Sanchez-Yague A, Will U, Tarantino I, et al. EUS-guided drainage of pancreatic fluid collections using a novel lumenapposing metal stent on an electrocautery-enhanced delivery system: a large retrospective study (with video). Gastrointest Endosc. 2015;82:1039-46.

19. Cremer M, Deviere J, Engelholm L. Endoscopic management of cysts and pseudocysts in chronic pancreatitis: long-term follow-up after 7 years of experience. Gastrointest Endosc. 1989:35:1-9.

20. Adler DG, Taylor $\sqcup$, Hasan R, Siddiqui AA. A retrospective study evaluating endoscopic ultrasound-guided drainage of pancreatic fluid collections using a novel lumen-apposing metal stent on an electrocautery enhanced delivery system. Endoscopic Ultrasound. 2017:6:389-93.

21. Sahar N, Kozarek R, Kanji ZS, Ross AS, Gluck M, Gan SI, et al. Do lumenapposing metal stents (LAMS) improve treatment outcomes of walled-off pancreatic necrosis over plastic stents using dual-modality drainage? Endosc Int Open. 2017;5:E1052-9.

22. Zhu H, Lin H, Jin Z, Du Y. Re-evaluation of the role of lumen-apposing metal stents (LAMS) for pancreatic fluid collection drainage. Gut. 2017;66: 2054-7.

\section{Ready to submit your research? Choose BMC and benefit from:}

- fast, convenient online submission

- thorough peer review by experienced researchers in your field

- rapid publication on acceptance

- support for research data, including large and complex data types

- gold Open Access which fosters wider collaboration and increased citations

- maximum visibility for your research: over $100 \mathrm{M}$ website views per year

At BMC, research is always in progress.

Learn more biomedcentral.com/submissions 